\title{
Current pharmacological treatment of heart failure: What is new in the 2021 ESC guidelines?
}

Heart failure (HF) is a complex of clinical symptoms and signs caused by structural and/ or functional abnormalities of the heart. The specific pathology underlying it determines subsequent treatment. The latest guidelines of the European Society of Cardiology (ESC) have been revised and structured according to the current classification of HF types, with the term "heart failure with mid-range ejection fraction" replaced by "heart failure with mildly reduced ejection fraction (HFmrEF).

The main purpose of this article is to briefly review the drug classes used in patients with HF. The presented guidelines primarily introduce a new, individualized approach to pharmacotherapy. In patients with heart failure and reduced left ventricular ejection fraction (HFrEF), pharmacotherapy is based on three main goals:

1. reduction in mortality;

2. to prevent re-hospitalization due to worsening HF;

3. improvement of the clinical, functional and quality of life condition.

In this group of patients, pharmacotherapy is the mainstay of treatment and should be initiated before other methods, including device therapy. So far, of course, heart failure has also been treated in relation to comorbidities, but now we have the opportunity and need to consider important clinical features when making therapeutic decisions. The intervention relates not only to the etiology of the disease, but also to important clinical elements such as blood pressure, heart rate, the presence of atrial fibrillation, the presence of chronic kidney disease, hyperkalemia, and finally the presence of congestion. A profiled look allows us to choose the available therapy in the best possible way.

The current standard of care for patients with HFrEF is the use of a drug that blocks or modifies the reninangiotensin- aldosterone (RAA) system, i.e. angiotensin-converting enzyme (ACEI) / angiotensin receptor -neprilysin inhibitor (ARNI), beta-blocker (BB), mineralocorticoid receptor antagonist (MRA) and a new class of drugs, sodium-glucose cotransporter 2 inhibitors (SGLT2i), irrespective of the coexistence of diabetes mellitus.

Among the latter, two molecules - dapagliflozin and empagliflozin - obtained registration and approval in HFrEF. The current guidelines still recommend the use of ARNI in patients who have persistent symptoms despite the combination of $\mathrm{ACEI}, \mathrm{BB}$ and MRA, however it is allowed to include ARNI in the first line instead of ACEI. Sartans (ARBs), another group of drugs that affect the function of the RAA system, retained their importance in patients intolerant to ACEI or ARNI.

Thus, there are four main classes of drugs that form the basis of HFrEF treatment. The concept of using these drugs is also new. Previous treatment options required titration, slow addition of subsequent groups of drugs and increasing doses until maximum values were reached. It took many months. The new guidelines propose a simultaneous entry with fundamental therapies, of course taking into account the patient's profile. Within a month, if successful, four groups of drugs needed to stop the progression of the disease should be added and only then doses should be titrated to the doses used in clinical trials, or, if this is impossible, to the maximum tolerated dose.

For the first time, the guidelines recommend pharmacotherapy in patients with HFmrEF. ACEI, BB, MRA and ARNI are in class of recommendation IIb, meaning "their use may be considered". In addition, the indications for the use of intravenous iron in a complex with carboxymaltose were extended with the recommendation to actively seek patients with iron deficiency. There is also a new molecule that appears for the first time in the therapeutic algorithm vericiguat (soluble guanylate cyclase stimulator), a drug with vasodilating effects, for patients at high risk of adverse events related to heart failure.

Address for correspondence: Adam Stanczyk, Department of Clinical Pharmacology. Ward of Internal Diseases Norbert Barlicki Memorial Teaching Hospital No1, Medical University of Lodz, Poland. Email: adam.stanczyk@umed.lodz.pl

Received: 12.09.2021 Accepted: 13.09.2021

Copyright $@ 2021$ Heart, Vessels and Transplantation

doi: $10.24969 /$ hvt.2020.274 
Unfortunately, the new European guidelines did not introduce any changes to the management of heart failure patients with preserved left ventricular ejection fraction (HFpEF). The guidelines still talk about the use of diuretics for symptoms of overhydration in these patients and about the optimal treatment of comorbidities, both cardiovascular and non-cardiovascular. However, there is still no dedicated pharmacotherapy for these patients

A detailed overview of the groups of drugs recommended for patients with heart failure is provided below and its recommendations in Table 2 .

ACEI: ACEI are first-line drugs to reduce mortality and morbidity, and to reduce symptoms.

$\mathrm{BB}$ : in combination with $\mathrm{ACEl}$ and a diuretic B-blocker therapy reduce mortality and morbidity, and ameliorate symptoms. BB should be initiated at least together with the ACEI and diuretic in clinically stable patients with euvolemia. In patients with acute heart failure, the initiation of therapy should be implemented in a hospital setting, with the dose being gradually increased to the recommended dose.

MRA: Spironolactone or eplerenone are indicated in combination with $A C E I$ and $B B$ in all patients to reduce mortality number of hospitalization and ameliorate symptoms. The use of eplerenone compared to spironolactone is associated with a significant lower percentage of induced gynecomastia.

ARNI: ARNI therapy in place of ACEI / ARB shows superiority in reducing the number of hospitalizations due to worsening $\mathrm{HF}$, cardiovascular and general mortality. Additional benefits include improving quality of life, reducing the progression of kidney disease and the risk of hyperkalaemia. ARNI also allow you to reduce the dose of diuretics used. A significantly more frequent complication of ARNI administration is symptomatic hypotension despite maintaining clinical benefits.

SGLT2 inhibitors: In the SGLT2i group, dapagliflozin and empagliflozin showed benefits of their use in patients with $\mathrm{HF}$ in combination with standard therapy, regardless of the diagnosis of diabetes. Diuretic-natriuretic properties bring additional benefits in terms of dose reduction of loop diuretics. The dark side of SGLT2 $i$ therapy is the increased risk of recurrent genital fungal infections.

Diuretics: Diuretics, especially loop diuretics, are recommended to alleviate symptoms. Their effect on mortality has not been investigated in randomized clinical trials. Their use improves exercise tolerance and reduces the number of hospitalizations. When selecting the appropriate dose, the diuretic effect of other drug groups (ARNI, MRA, SGLT2i) should be taken into account.

If- channel inhibitor: Ivabradine is the only representative of this group of drugs. Ivabradine slows the heart rate by affecting the sinus node and therefore its use should be limited to patients with sinus rhythm $\geq 70$ beats/min who cannot tolerate or has contraindications to $\mathrm{BB}$, or has heart rate $\geq 70$ beats/min despite optimal BB dose. In symptomatic patients with HFrEF, after joining the standard therapy, Ivabradine reduces cardiovascular mortality and the number of hospitalizations. It should be remembered that before starting Ivabradine, the target dose of BB should be reached.

Digoxin: Digoxin has a very narrow therapeutic window, therefore serum levels should be monitored regularly. In the group of patients with HFrEF and sinus rhythm, digoxin reduces the risk of hospitalization, however, the routine effect of combined therapy with BB has not been assessed. Despite conflicting results regarding the effect on mortality in patients with $\mathrm{HF}$ and atrial fibrillation in symptomatic patients, digoxin may be used to slow down the high ventricular rate.

New groups of drugs studied in the HF patients are Vericiguat and Omecamtiv mecarbil. Vericiguat is an oral soluble guanylate cyclase receptor stimulator. According to the results of VICTORIA study (2) it may be considered, in addition to standard therapy for $\mathrm{HFrEF}$, to reduce the risk of cardiovascular mortality and hospitalizations for HF. Omecamtiv mecarbil is a selective activator of myosin in the heart and studies to date have shown an improvement in cardiac function in patients with HFrEF (GALACTIC-HF study) (3). In the future it may be added to standard therapy to reduce the risk of hospitalization and cardiovascular mortality.

\section{Conclusions:}

The current guidelines are a breakthrough in the approach to heart failure not only because of new therapies, but mainly because of the approach to treatment. The newest recommendations mark the beginning of an era in which personalization of implemented therapies comes to the fore. Targeted management of the group of patients discharged from the hospital is also extremely important. It is made clear that patients should be discharged with effective, optimal, oral treatment. It should also be considered whether there is a need to implement iron supplementation. 
During a short-term follow-up, the physician should check the patient's condition, pharmacological treatment should be quickly optimized and modified.

Adam Stanczyk, Department of Clinical Pharmacology. Ward of Internal Diseases

Norbert Barlicki Memorial Teaching Hospital No 1, Medical University of Lodz, Poland.

Peer-review: Internal Conflict of interest: None to declare Authorship: A.S. Acknowledgement and funding: None to declare

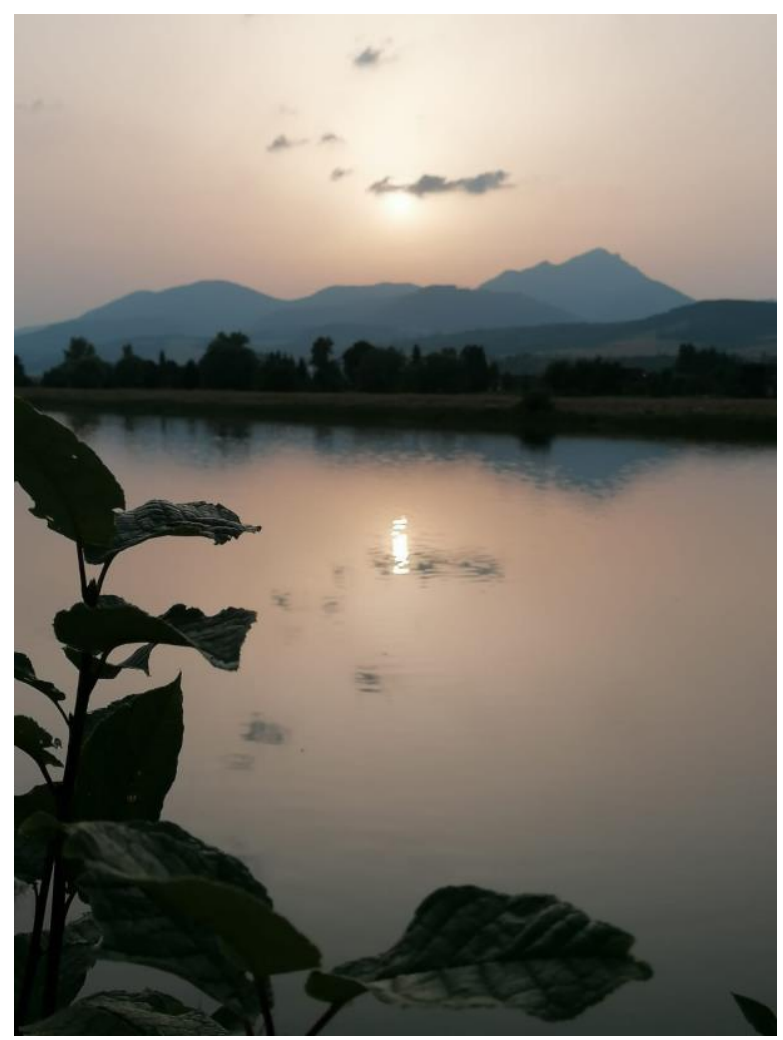

\section{References}

1.McDonagh TA, Metra M, Adamo M, Gardner RS, Baumbach A, Bohm M, et al. ESC Scientific Document Group, 2021 ESC Guidelines for the diagnosis and treatment of acute and chronic heart failure: Developed by the Task Force for the diagnosis and treatment of acute and chronic heart failure of the European Society of Cardiology (ESC) With the special contribution of the Heart Failure Association (HFA) of the ESC. Eur Heart J 2021; ehab368, doi: 10.1093/eurheartj/ehab368

2.Armstrong PW, Pieske B, Anstrom KJ, Ezekowitz J, Hernandez AF, Butler J, et al. VICTORIA Study Group. Vericiguat in patients with heart failure and reduced ejection fraction. N Engl J Med 2020; 382: 1883-93.

3.Teerlink JR, Diaz R, Felker GM, McMurray JJV, Metra $\mathrm{M}$, Solomon SD, et al. GALACTIC-HF Investigators. Cardiac myosin activation with omecamtiv mecarbil in systolic heart failure. N Engl J Med 2021; 384: 105-16.

Sunset reflection in the Vah river, Liptov region, Slovakia. Ljuba Bacharova, Bratislava, Slovakia. 